\title{
COAXIAL TO WAVEGUIDE TRANSITIONS AND DEVICE UNDER TEST CHARACTERIZATION BY MEANS OF INVERSE TECHNIQUES
}

\begin{tabular}{|r|l|}
\hline Journal: & Microwave and Optical Technology Letters \\
\hline Wanuscript ID: & MOP-09-1073 \\
\hline Datey - Manuscript type: & Research Article \\
\hline Complete List of Authors: & $\begin{array}{r}\text { Lozano-Guerrero, Antonio; Universidad Politécnica de Cartagena, } \\
\text { Departamento de Tecnologías de la Información y las } \\
\text { Comunicaciones } \\
\text { Monzo-Cabrera, Juan; Universidad Politécncia de Cartagena, } \\
\text { Tecnologías de la Información y las Comunicaciones } \\
\text { Clemente-Fernández, Francisco; Universidad Politécnica de } \\
\text { Cartagena, Tecnologías de la Información y las Comunicaciones } \\
\text { Pedreño-Molina, J.L.; Universidad Politecncia de Cartagena, } \\
\text { Tecnologías de la Información y Comunicaciones } \\
\text { Diaz-Morcillo, Alejandro; Polytechnic University of Cartagena, } \\
\text { Information Technologies and Communicactions }\end{array}$ \\
\hline Keywords: & $\begin{array}{l}\text { Coaxial to waveguide transitions, device under test, inverse } \\
\text { techniques, two-tier calibration, genetic algorithms }\end{array}$ \\
\hline \hline
\end{tabular}

\section{ScholaroNE Manuscript Central}




\title{
COAXIAL TO WAVEGUIDE TRANSITIONS AND DEVICE UNDER TEST CHARACTERIZATION BY MEANS OF INVERSE TECHNIQUES
}

\section{Antonio José Lozano-Guerrero, Juan Monzó-Cabrera, Francisco Javier Clemente- Fernández, Juan Luis Pedreño-Molina, Alejandro Díaz-Morcillo}

Departamento de Tecnologías de la Información y las Comunicaciones

Universidad Politécnica de Cartagena

Cartagena, Spain

email: antonio.lozano@upct.es

Fax: +34 968325973

\begin{abstract}
A new two-tier inverse characterization technique for coaxial to waveguide transitions including a device under test is presented in this paper. The transitions and the device under test are characterized by its scattering parameters and a cascade procedure is used in order to compare calculations and measurements during the unterminating procedure. Two different standard types such as short-circuits and thrus are used, and the two transitions jointly with the device under test are simultaneously characterized. Genetic algorithms and a gradient based method have been used for error minimization during the unterminating stage. Results of this two-tier inverse technique are compared to those provided by measurements, simulations and the three-cavity method, showing that it is possible to properly characterize the coaxial to waveguide transitions and the device under test in a flexible and accurate way.
\end{abstract}

Key words: Coaxial to waveguide transitions; device under test; inverse techniques; two-tier calibration; genetic algorithms 


\section{INTRODUCTION}

Transitions provide the necessary interconnection capability between different types of transmission lines such as waveguides, coaxial, coplanar or microstrip lines. A good characterization of these elements is necessary in order to assess their behavior and to carry out design and optimization processes.

Coaxial to waveguide transitions have been widely analyzed [1-4] since they are used in a multitude of microwave applications: multiplexers, diplexers, power dividers, and transitions (which can also be viewed as a particular case of T-junctions).

If the scattering parameters of the employed transitions are known they can be removed from vector network analyzer (VNA) measurements by the so-called de-embedding process [5-6]. A precise evaluation of any transition becomes essential then to characterize a device under test (DUT). However, obtaining the transition parameters is not immediate as they must be characterized from measurements made at the VNA reference plane where known standards are evaluated. This process is referred to as unterminating [6].

Numerous methods and techniques have been developed using different approaches. Iterative approaches have been studied showing that it is possible to use redundant standards to increase the accuracy improving the conventional calibration procedures [6-7]. Genetic algorithms have been employed to de-embed the test fixture effect using a thru measurement in [8] without using any other standards. A previous study of this work in [9] uses genetic algorithms and a gradient based method to extract $S$-parameters from coaxial to waveguide transitions without taking into account the device under test to be measured. CAD models of transitions have been used in coaxial to waveguide transitions [10]. However, a full-wave electromagnetic analysis of these structures requires frequency-dependent and time-consuming calculations. Additionally, specific characteristics of the transitions cannot be taken into account sometimes due to the high complexity of the structure under study. To overcome these drawbacks some characterization methods based on $S$-parameter measurements have been presented, specifically focused on coaxial probe modeling in waveguides and cavities. One of the most important contributions of probe-excited waveguide problem was presented in [1], where a rigorous method to obtain the 2-port scattering matrix of a probe-excited semiinfinite waveguide is shown. This procedure, known as the three cavities moment method, uses three cavities and their input reflection coefficients for obtaining three linear equations. The main drawback of this method is a restriction regarding the phases of the reflection coefficient of the short-circuited waveguide sections, which must not have $360^{\circ}$ differences at a given frequency. This procedure assumes only one propagating mode and an extension is presented in [3]. Further research on the three cavities approach can be found in [4] where a coaxial to a rectangular waveguide junction is analyzed using the five-cavity moment method in combination with network cascading techniques and an interpolation method. All these methods use exclusively waveguide short-circuit standards at different electrical lengths from the transition in order to extract its behavior versus frequency.

In this work, two different standard types such as short-circuits and thrus are used and two transitions are simultaneously characterized. A new inverse characterization technique is introduced in order to evaluate coaxial to waveguide transitions jointly with the device under test. This novel unterminating procedure is carried out by minimizing the error between calculations of scattering matrix concatenations that contain the 
transitions and the DUT as unknown parameters and measurements of several structures that reproduce the calculated scenarios. The obtained results for the transition inverse characterization are compared to those provided by the three cavities method, whereas measurements and simulations are used for the DUT validation.

\section{THEORY}

An inverse technique [11] provides the estimation of an unknown parameter by comparing the experimental response of the analysed structure to the calculated simulation of the experimental scenario. In this study the two-port scattering matrices $(S)$ of two slightly different coaxial to waveguide transitions and a device under test are simultaneously obtained using an inverse procedure. The proposed procedure can be however extended to any other transition and line types. In Figure 1 a scheme of the WR-340 transitions under study and the device under test is depicted. As it can be observed, these transitions include several tuning screws in order to obtain good matching levels within the operating bandwidth. The device under test consists of a 2 cm WR-340 waveguide holder whose cross section is completely filled with a $1.043 \mathrm{~cm}$ PTFE slab.

\subsection{Parameter Description}

The two-port scattering parameters of each coaxial to waveguide transition and those of the device under test are modelled through their magnitude and phase representation. This leads to 18 different unknown real parameters when considering the fact that $S_{12}=S_{21}$ due to reciprocity. Each scattering parameter will be evaluated at different frequency values within the studied bandwidth. The $S$ matrices for each transition and the device under test are defined as

$$
\begin{aligned}
& {\left[\begin{array}{ll}
S_{11}^{t r 1} & S_{12}^{t r 1} \\
S_{21}^{t r 1} & S_{22}^{t r 1}
\end{array}\right]=\left[\begin{array}{ll}
A e^{j \phi_{1}} & B e^{j \phi_{2}} \\
B e^{j \phi_{2}} & C e^{j \phi_{3}}
\end{array}\right]} \\
& {\left[\begin{array}{ll}
S_{11}^{t r 2} & S_{12}^{t r 2} \\
S_{21}^{t r 2} & S_{22}^{t r 2}
\end{array}\right]=\left[\begin{array}{ll}
D e^{j \phi_{4}} & E e^{j \phi_{5}} \\
E e^{j \phi_{5}} & F e^{j \phi_{6}}
\end{array}\right]} \\
& {\left[\begin{array}{ll}
S_{11}^{D U T} & S_{12}^{D U T} \\
S_{21}^{D U T} & S_{22}^{D U T}
\end{array}\right]=\left[\begin{array}{ll}
G e^{j \phi_{7}} & H e^{j \phi_{8}} \\
H e^{j \phi_{8}} & I e^{j \phi_{9}}
\end{array}\right]}
\end{aligned}
$$

where $\operatorname{tr} 1$ and $\operatorname{tr} 2$ refer to each transition and DUT is the device under test. $A, B, C, D$, $E, F, G, H, I \in[0,1]$ represent the magnitude of the parameters and $\phi_{1}, \phi_{2}, \phi_{3}, \phi_{4}, \phi_{5}, \phi_{6}, \phi_{7}$, $\phi_{8}, \phi_{9} \in[-\pi, \pi]$ are their phase values. Employed coaxial to waveguide transitions are slightly different and the proposed method deals with their particular differences providing an accurate set of $S$-parameters for each one. 


\subsection{Cascade Procedure}

As stated before short-circuits and thrus with different lengths have been used in this work during the two-tier calibration process. All the employed standards during the extracting process can be seen in Figure 2. Represented shorts for Port 1 have also been implemented for Port 2. The connection of both standards' types to the coaxial to waveguide transition and to the device under test can be interpreted as a cascade of devices. The parameters for the transmission line matrix are then defined as

$$
\left[S_{t r x l i n e}\right]=\left[\begin{array}{cc}
0 & e^{-j \beta l} \\
e^{-j \beta l} & 0
\end{array}\right]
$$

where $\beta$ is the phase constant in the waveguide and $l$ is the length of the line. The cascade formulas for $S$-parameters are well known and can be found in literature [13].

\subsection{Optimization Techniques}

The values of $S_{i j}^{t r n}$ and $S_{i j}^{D U T}(n=1,2$ and $i, j=1,2)$ are sequentially obtained for 601 frequency points in the range under study with the help of two different optimization techniques. For the first frequency point, the initial solution is obtained with the aid of a genetic algorithms tool implemented in Matlab [14]. A more accurate refinement is then obtained with the aid of a gradient based optimization method using as initial point for the search procedure the best solution obtained in the genetic algorithms' stage. The solution of the following frequency point is calculated with the gradient based method by using as initial point the solution of the preceding frequency point iteratively. A steep deviation in the solution frequency response may lead to wrong solution values increasing the fitness function evaluation. In this case a genetic algorithms stage is generated again for the wrong last frequency point calculation.

The flowchart of the optimization procedure employed in this work is shown in Figure 3. Genetic algorithms have been chosen in this work to find the global minimum error at the first optimization stage. An individual of genetic algorithms represents a possible solution of our problem that contains the estimation of 18 different unknown real parameters necessary to characterize the two transitions and the device to be measured. These 18 unknown parameters require a high number of individuals and generations to reach a good approximation. Thus, 300 generations, 150 individuals, 120 crossovers per generation and 5 mutations per generation have been used during the genetic algorithms' stage. In the first generation a random initial population is evaluated. Crossover and mutation operations define the following generations evolving towards the optimum individual (our solution).

The gradient based optimization stage uses a multivariable Matlab® function that implements the Quasi-Newton method.

Both optimization algorithms require an evaluation function that becomes a crucial aspect. In this work, the evaluation function takes into account the magnitude of the difference between the measured complex scattering parameters $\left(S_{i j}^{m}\right)$ and the calculated 
ones $\left(S_{i j}^{c}\right)$ for different experimental set ups.

The expression to evaluate the error when using any of the three unknown devices including a short-circuit standard during the optimization procedure is shown in (5). To evaluate a thru connection between transitions including or not the device under test and a $12.65 \mathrm{~cm}$ transmission line eq. (6) is used. The generalised evaluation function expression including all the error contributions is shown in (7). The evaluation is carried out for each frequency point.

$$
\begin{gathered}
f_{\text {short }}=\left|S_{11}^{m}-S_{11}^{c}\right|^{2} \\
f_{\text {thru }}=\sum_{i=1}^{2} \sum_{j=1}^{2}\left|S_{i j}^{m}-S_{i j}^{c}\right|^{2} \\
f=\sqrt{f_{\text {thru } 1}+f_{\text {thru } 2}+\cdots+\cdots+f_{\text {thrun }}+f_{\text {short } 1}+f_{\text {short } 2}+\cdots+f_{\text {shortn }}}
\end{gathered}
$$

\section{EXPERIMENTAL SET UP}

A Rohde \& Schwarz ZVM vector network analyzer (VNA) has been used in this work in order to measure the scattering matrix frequency behavior of the coaxial to waveguide transitions when using different standards. 601 frequency points were collected in each measurement in the 2.2-2.8 GHz frequency range. Therefore, it was ensured that WR-340 waveguide components worked only with the $\mathrm{TE}_{10}$ main mode.

A Rohde \& Schwarz ZV-Z32 PC 3.5 fixed matched calibration kit was employed in order to calibrate the VNA at the calibration plane shown in Figure 1. The coaxial to waveguide transitions used in this work belong to a Continental Microwave WCK340HP waveguide calibration kit [15].

\section{RESULTS}

Due to the high number of employed standards a time of approximately 25 minutes has been necessary to carry out the inverse procedure in a personal computer with 3 GByte RAM memory and a $2 \mathrm{GHz}$ processor. A minimum number of standards is required to obtain a valid solution and extra standards can be used to provide redundancy and increase the accuracy of the results [12].

Figures 4 and 5 show the results for the transition 1 for both the magnitude and phase of $S_{11}$ and $S_{12} S_{21}$ product when all short-circuits and thrus shown in Figure 2 are used to evaluate the fitness function. The results of this new inverse technique are compared to those provided by the three cavities method for both transitions. From these results it can be appreciated that both techniques show good agreement. An absolute average error of 0.0123 has been obtained for the $S_{11}$ magnitude and 0.1318 rad. for the phase when comparing with the three cavities method in the frequency range under study. $S_{12} S_{21}$ product has been included in this work in order to be able to compare our results to the three cavities technique described in [1]. $S_{12} S_{21}$ provides better results as an absolute average error of 0.0017 has been obtained for the magnitude and $0.1034 \mathrm{rad}$. for the phase. $S_{22}$ offers similar results than $S_{11}$ and consequently they have not been depicted.

In Figures 6 and 7 results for the $S$-parameters (magnitude and phase) of the device 
under test are depicted. Calculated results with the proposed technique are compared to those experimental results obtained by using a waveguide calibration kit [15] and to those simulated with the commercial software CST Microwave Studio [16]. To model the PTFE material the values $\varepsilon^{\prime}{ }_{r}=2.05$ and $\tan \delta=0.0002$ were included in the simulations. An absolute average error of 0.0077 has been obtained for the $S_{11}$ magnitude and $0.0263 \mathrm{rad}$. for the phase when comparing with the waveguide calibration measurements in the frequency range under study. $S_{21}$ provides an absolute average error of 0.0039 for the magnitude and $0.0243 \mathrm{rad}$. for the phase.

Good agreement can be found for the three techniques (inverse procedure, simulations and DUT experimental measurements with waveguide calibration) showing that the proposed procedure can be successfully employed to obtain the coaxial to waveguide transitions and the device under test $S$-parameters.

\section{CONCLUSION}

A new two-tier inverse technique for characterizing jointly two coaxial to waveguide transitions and a device under test based on the use of genetic algorithms and a gradient based method has been described and compared to the three-cavity measurement technique, measurements and simulations. Very good results are obtained by using several calibration standards such as short-circuits and thrus.

This inverse technique provides very similar results to those obtained by the threecavity method or simulations but, additionally, is able to handle any standards combination provided that they can be properly included in $S$ parameter cascade calculations. Additionally, the proposed technique is able to provide an accurate characterization of the device under test without the need of de-embedding techniques, which cannot be done with the three-cavity method.

Although applied to coaxial to waveguide transitions, this inverse technique can be extended to other transition types and, therefore, further research is envisaged in that direction.

\section{ACKNOWLEDGEMENTS}

This work was supported in part by the Ministerio de Ciencia e Innovación under the project with reference CIT-020000-2008-43. 


\section{REFERENCES}

1. J. F. Liang, H. Chang, and K. A. Zaki, Coaxial probe modeling in waveguides and cavities, IEEE Trans Microwave Theory Tech 40 (1992), 2172-2180.

2. M. Hajian, D. P. Tran, and L. P. Ligthart, Modeling the transition between a coaxial line and a flat rectangular waveguide, Ninth International Conference on Antennas and Propagation (1995), 269-272.

3. Hui-Wen Yao and K. A. Zaki, Modeling generalized coaxial probes in rectangular waveguides, IEEE MTT-S Int Microwave Symp Dig 2 (1995), 979982.

4. Y. F. Huang, H. David, S. Raymond, and P. Protap, Modeling of cavity-backed coaxial line to rectangular waveguide junction, Int J RF Microwave Comput Aided Eng 8 (1998), 20-26.

5. R.F. Bauer and P. Penfield, De-embedding and unterminating, IEEE Trans Microwave Theory Tech 22 (1974), 282-288.

6. D. Williams, De-embedding and unterminating microwave fixtures with nonlinear least squares, IEEE Trans Microwave Theory Tech 38 (1990), 787791.

7. R.B. Marks, A multiline method of analyzer calibration, IEEE Trans Microwave Theory Tech 39 (1991), 1205-1215.

8. A. S. Adalev, N. V. Korovkin, M. Hayakawa, and J. B. Nitsch, Deembeding and unterminating microwave fixtures with the genetic algorithm, IEEE Trans Microwave Theory Tech 54 (2006), 3131-3140

9. A. J. Lozano-Guerrero, J. Monzó-Cabrera, F. J. Clemente-Fernández, J. L. Pedreño-Molina, and A. Díaz-Morcillo, Precise coaxial to waveguide characterization by means of inverse techniques, IEEE Trans Microwave Theory Tech (under review).

10. F. Arndt, I. Ahrens, U. Papziner, U. Wiechmann, and R. Wilkeit, Optimized Eplane T-junction series power dividers, IEEE Trans Microwave Theory Tech 35 (1987), 1052-1059.

11. M. E. Requena-Pérez, A. Albero-Ortiz, and J. Monzó-Cabrera, Combined use of genetic algorithms and gradient descent optimization methods for accurate inverse permittivity measurement, IEEE Trans Microwave Theory Tech 54 (2006), 615-624.

12. D.F. Williams, J.C.M. Wang, and U. Arz, An optimal vector-network-analyzer calibration algorithm, IEEE Trans Microwave Theory Tech 51 (2003), 23912401.

13. K. Rothemund, H. W Glock, and U. Rienen, Eigenmode calculation of complex RF-structures using S-parameters, IEEE Trans Magn 36 (2000), 1501-1503.

14. C. R. Houck, J. A. Joines, and M. G. Kay, A genetic algorithm for function optimization: A MATLAB implementation, The Mathworks, Natick, MA, NCSU-IE TR 95-09, (1995).

15. Waveguide Component Specifications and Design Handbook. Edition \#7. Continental Microwave Division. Available at www.cobham.com.

16. www.cst.com 


\section{LIST OF CAPTIONS}

Figure 1 Set up for coaxial to waveguide transitions and device under test

Figure 2 Thrus and shorts employed to obtain the coaxial to waveguide and DUT $S$ parameter matrices. Equivalent shorts have been used for port 2

Figure 3 Flowchart of the procedure

Figure $4 S$-parameter magnitude for coaxial to waveguide transition 1

Figure $5 S$-parameter phase for coaxial to waveguide transition 1

Figure $6 S$-parameter magnitude for the device under test

Figure $7 \quad S$-parameter phase for the device under test 


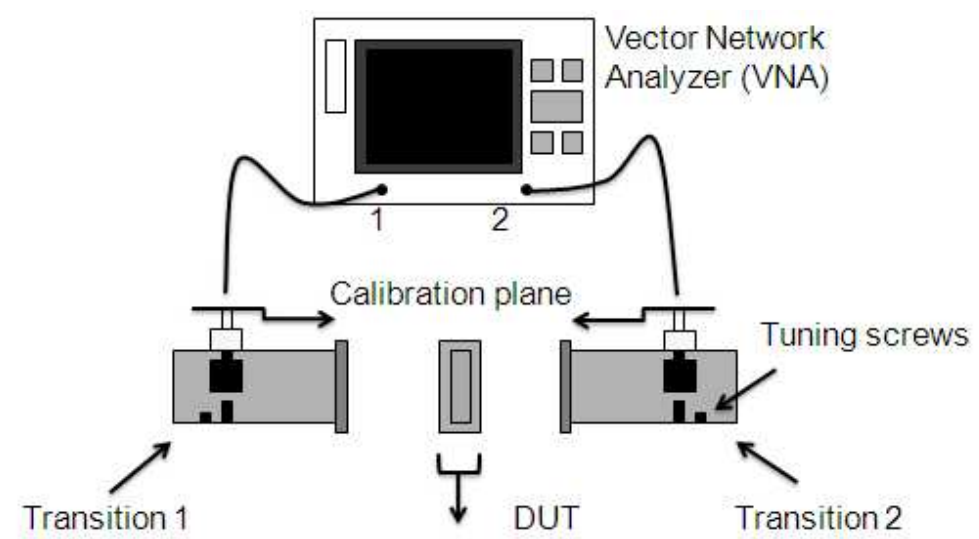

$176 \times 132 \mathrm{~mm}(96 \times 96 \mathrm{DPI})$ 


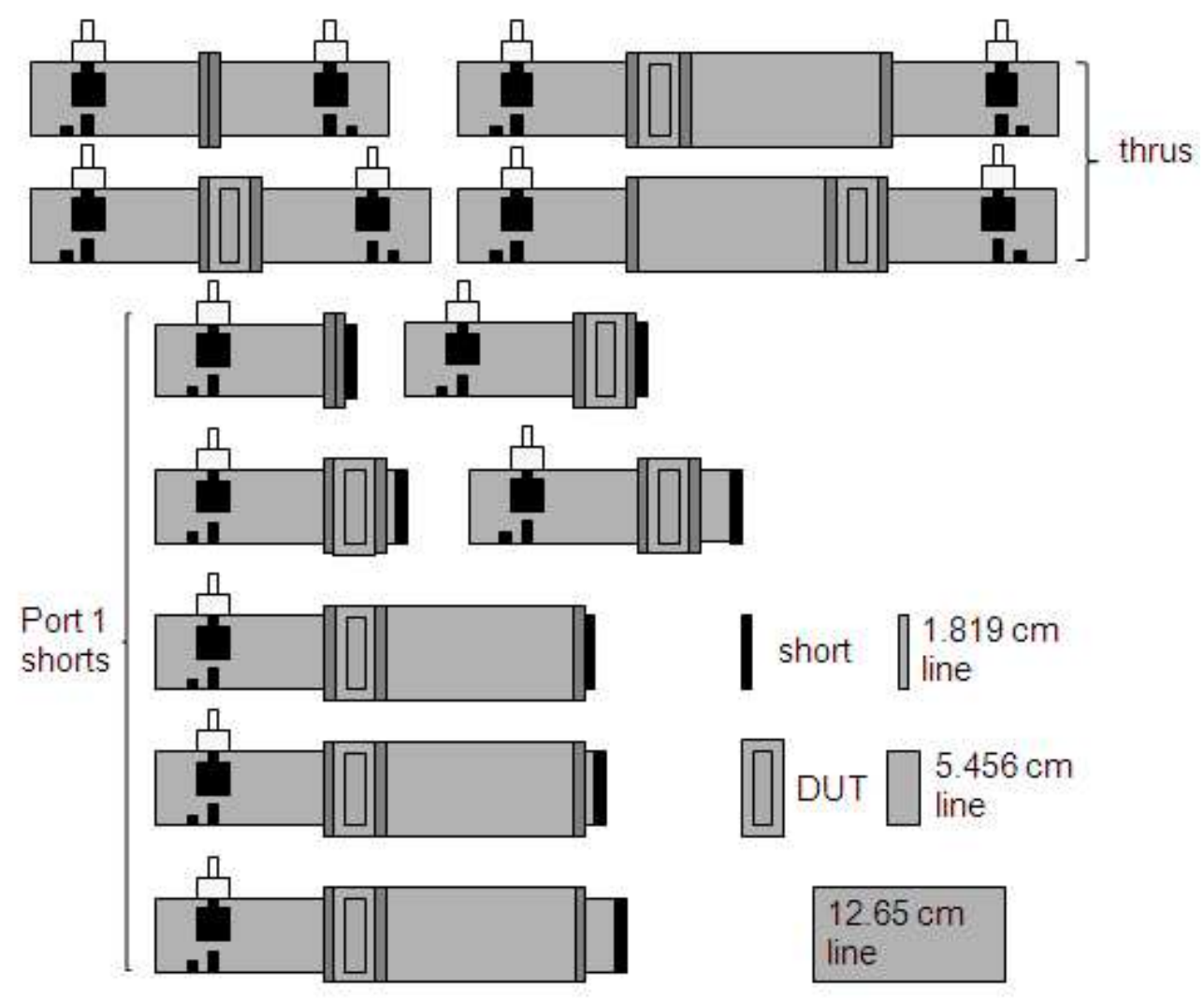

$139 \times 120 \mathrm{~mm}(96 \times 96 \mathrm{DPI})$ 


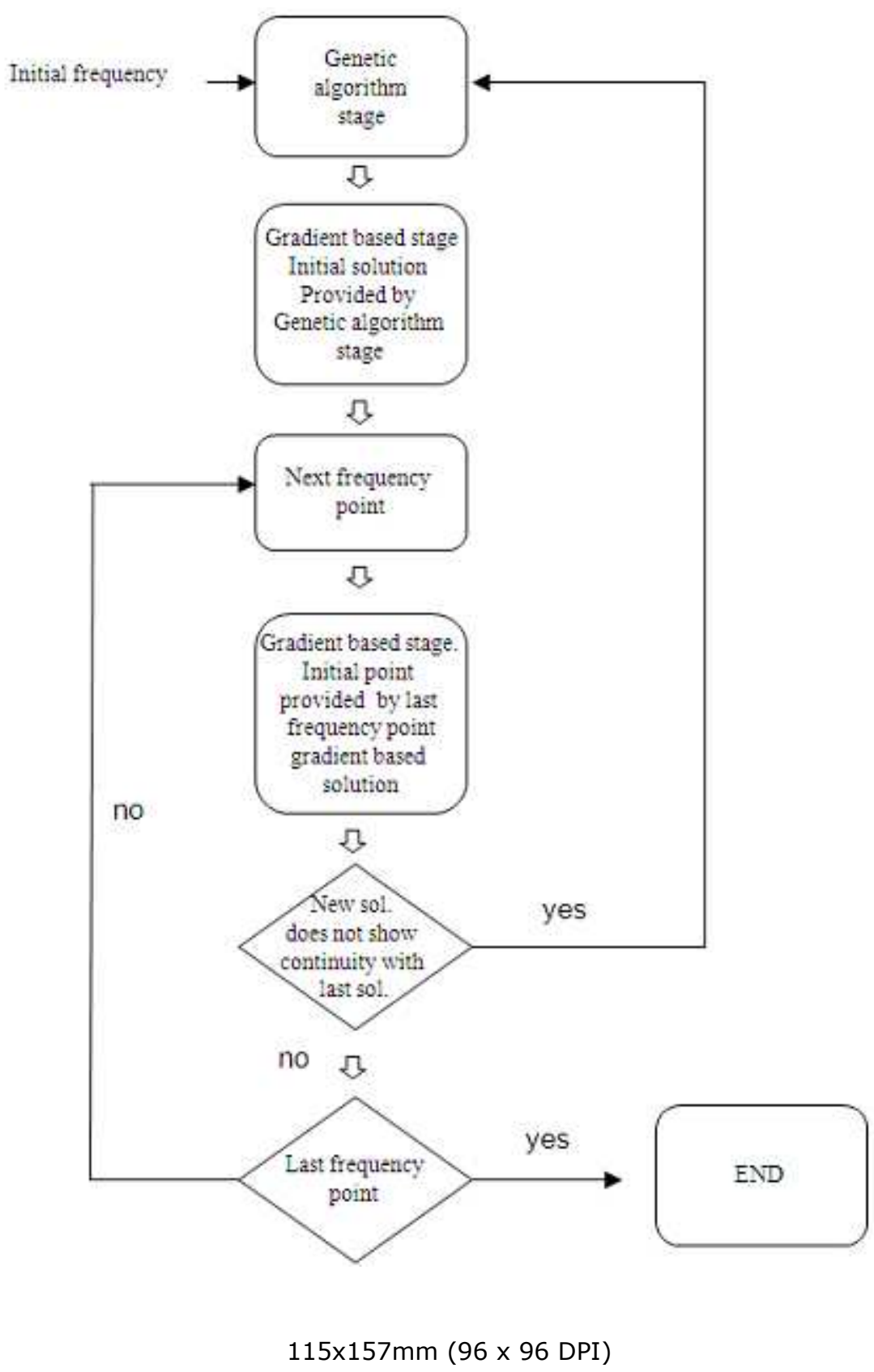




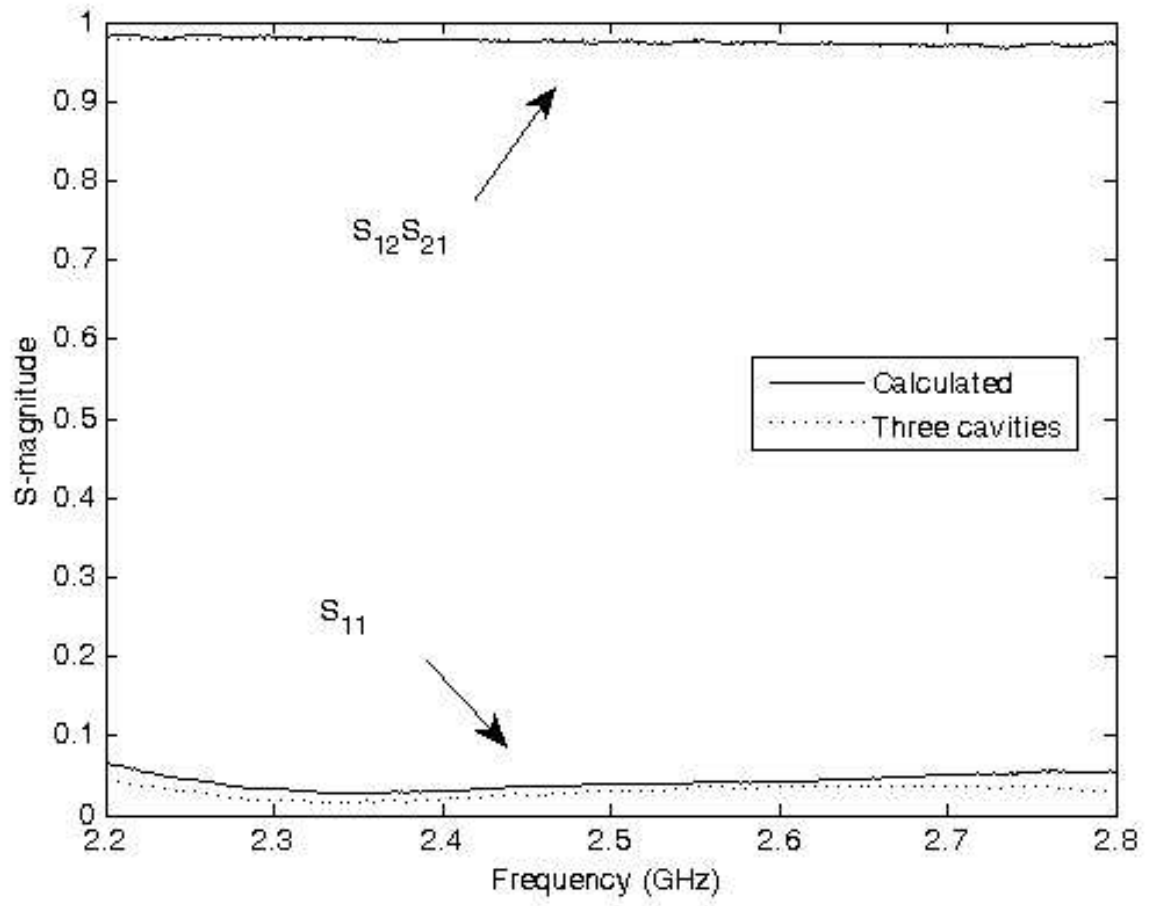

$148 \times 111 \mathrm{~mm}(96 \times 96 \mathrm{DPI})$

John Wiley \& Sons 
1

2

3

4

5

6

7

8

10

11

12

14

15

16

17

18

19

20

21

22

23

24

25

26

27

28

29

30

31

32

33

34

35

36

37

38

39

40

41

42

43

44

45

46

47

48

49

50

51

52

53

54

55

56

57

58

59

60

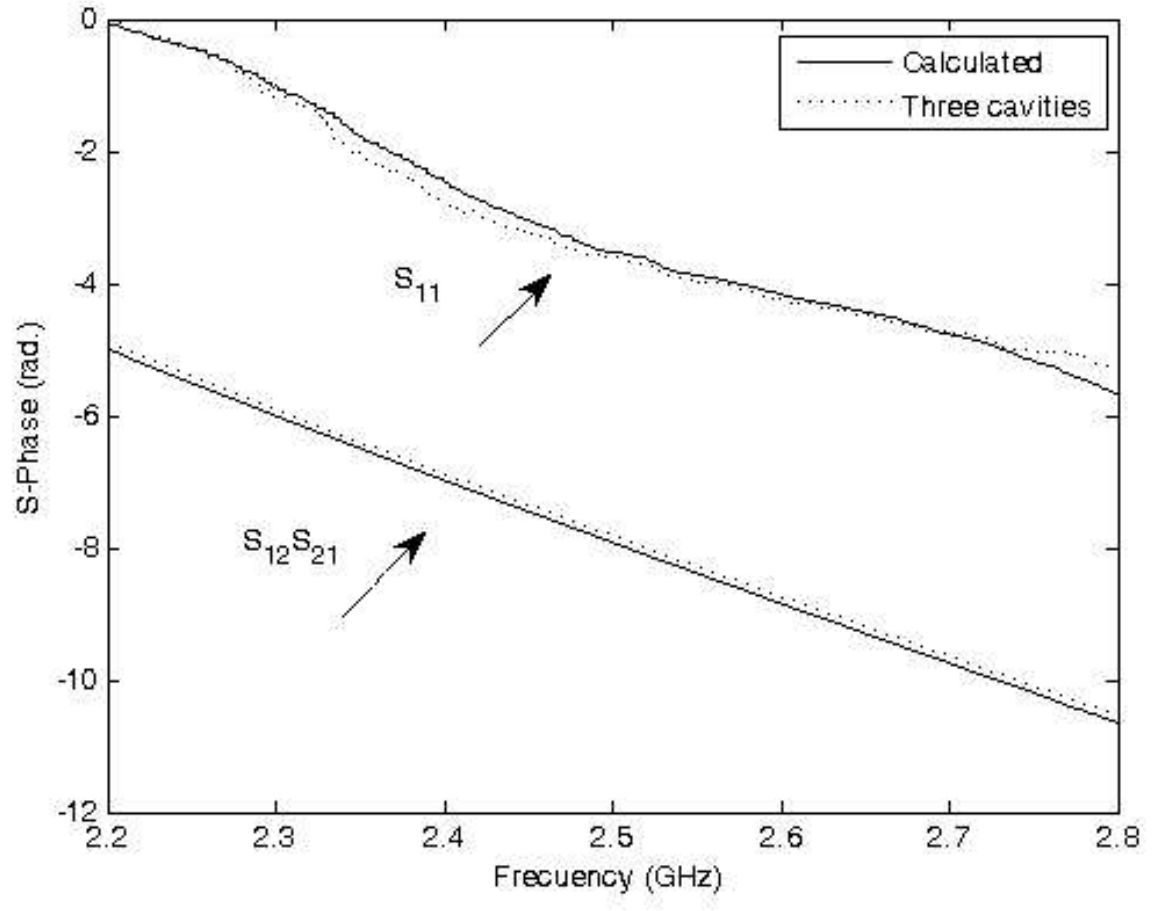

$148 \times 111 \mathrm{~mm}(96 \times 96 \mathrm{DPI})$ 


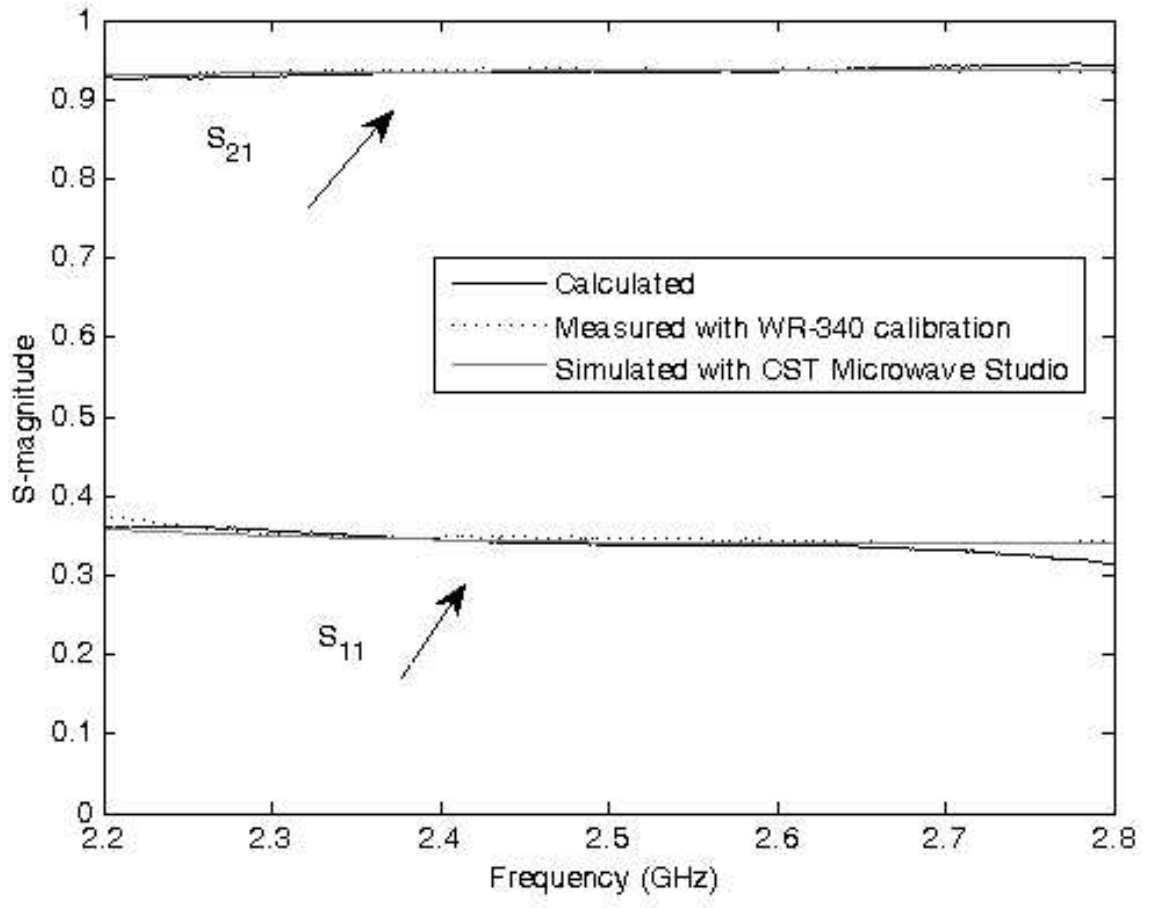

$148 \times 111 \mathrm{~mm}(96 \times 96 \mathrm{DPI})$ 
1

2

3

4

5

6

7

8

10

11

12

13

14

15

16

17

18

19

20

21

22

23

24

25

26

27

28

29

30

31

32

33

34

35

36

37

38

39

40

41

42

43

44

45

46

47

48

49

50

51

52

53

54

55

56

57

58

59

60

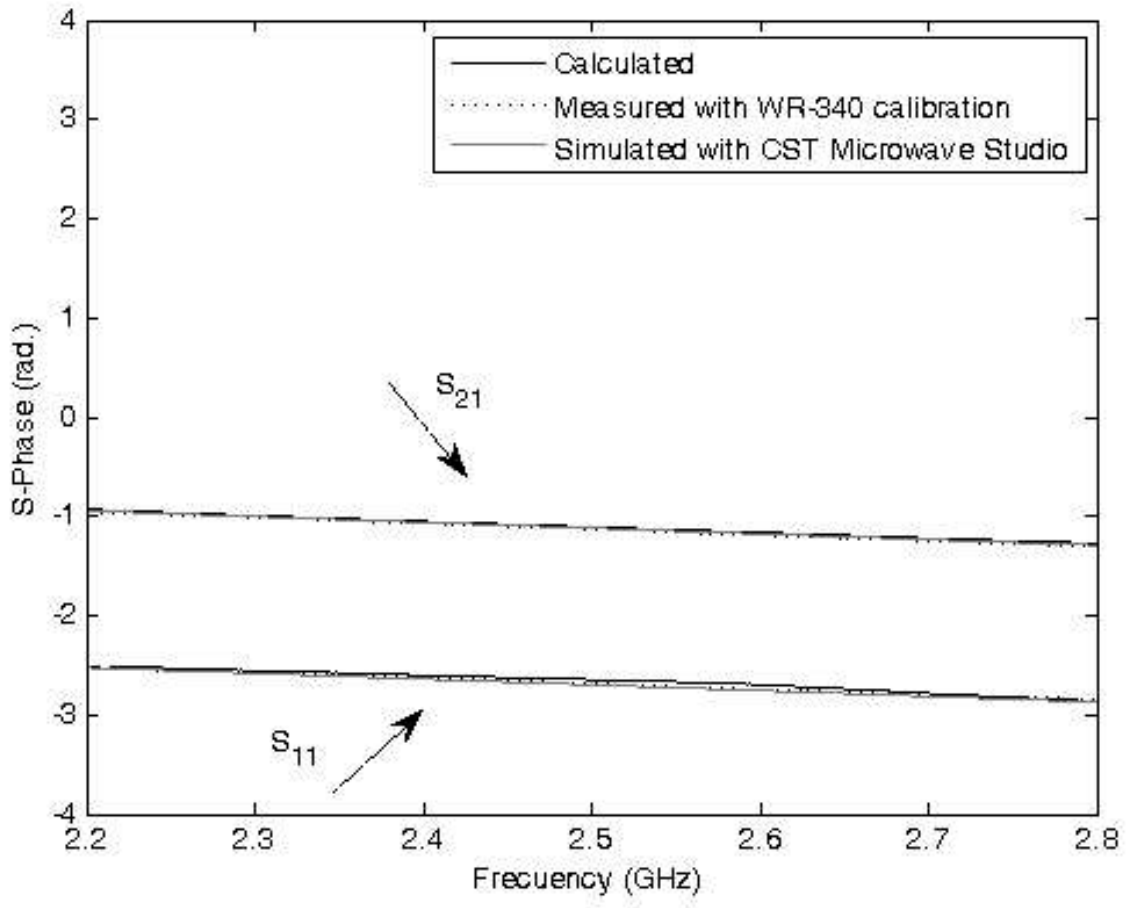

$148 \times 111 \mathrm{~mm}(96 \times 96 \mathrm{DPI})$ 